\title{
Coenzyme Q10, oxidative stress markers, and sperm DNA damage in men with idiopathic oligoasthenoteratospermia
}

\author{
Ahmed T Alahmar ${ }^{1}$, Pallav Sengupta ${ }^{2}$, Sulagna Dutta ${ }^{2}$, Aldo E. Calogero ${ }^{3}$ \\ 'Department of Medical Physiology, College of Medicine, University of Babylon, Hillah, Iraq; ${ }^{2}$ Department of Physiology, MAHSA University, Selangor, \\ Malaysia; ${ }^{3}$ Division of Andrology and Endocrinology, University Teaching Hospital "Policlinico-Vittorio Emanuele”, University of Catania, Catania, Italy
}

Objective: Oxidative stress (OS) plays a key role in the etiology of unexplained male infertility. Coenzyme Q10 (CoQ10) is a potent antioxidant that may improve semen quality and OS in infertile men with idiopathic oligoasthenoteratospermia (OAT), but the underlying mechanism is unknown. Therefore, the present study was undertaken to investigate the effect of CoQ10 on OS markers and sperm DNA damage in infertile patients with idiopathic OAT.

Methods: This prospective controlled study included 50 patients with idiopathic OAT and 50 fertile men who served as controls. All patients underwent a comprehensive medical assessment. Patients and controls received $200 \mathrm{mg}$ of oral CoQ10 once daily for 3 months. Semen and blood were collected and analyzed for sperm parameters, seminal CoQ10 levels, reactive oxygen species (ROS) levels, total antioxidant capacity, catalase, sperm DNA fragmentation (SDF), and serum hormonal profile.

Results: The administration of CoQ10 to patients with idiopathic OAT significantly improved sperm quality and seminal antioxidant status and significantly reduced total ROS and SDF levels compared to pretreatment values.

Conclusion: CoQ10, at a dose of $200 \mathrm{mg} /$ day for 3 months, may be a potential therapy for infertile patients with idiopathic OAT, as it improved sperm parameters and reduced OS and SDF in these patients.

Keywords: Coenzyme Q10; Male infertility; Oxidative stress; Sperm DNA fragmentation

\section{Introduction}

Infertility is defined as the failure of conception after at least 1 year of regular unprotected sexual intercourse [1]. The prevalence of infertility among reproductive-aged couples is approximately $8 \%-$ $15 \%$ [2], with male factor infertility accounting for $50 \%$ of cases [3].

Received: September 13, 2020 · Revised: December 10, 2020 · Accepted: December 11,2020

Corresponding author: Ahmed T Alahmar

Department of Medical Physiology, College of Medicine, University of Babylon, 88 University St, Hillah, Babyl 50001, Iraq

Tel: +96-47808180900 E-mail: ahmed.t.alahmar@gmail.com

This is an Open Access article distributed under the terms of the Creative Commons Attribution Non-Commercial License (http://creativecommons.org/licenses/by-nc/4.0/) which permits unrestricted non-commercial use, distribution, and reproduction in any medium, provided the original work is properly cited.
Infertility in men has been linked to endocrine disorders, developmental anomalies, systemic diseases, and environmental, immunological, and genetic factors [4-6]. However, in approximately $25 \%$ of infertile men, no cause can be identified for semen abnormalities; this condition is referred to as idiopathic infertility [7].

Oxidative stress (OS) has been reported as a key factor contributing to idiopathic male infertility [8]. OS may occur as a consequence of higher ratios of oxidants (free radicals and/or reactive oxygen species [ROS]) to antioxidants in the seminal plasma [9]. The etiology of OS can be attributed to several intrinsic or extrinsic factors, including ageing, varicocele, infection, cryptorchidism, testicular torsion, radiotherapy, chemotherapy, and toxins $[10,11]$. Specific physiological functions, such as sperm capacitation, the acrosome reaction, and fertilization require ROS [6,12]. Nonetheless, overproduction of ROS may impair sperm membrane and DNA integrity, resulting in de- 
creased sperm membrane fluidity and changes in the fertilizing capability of sperm [13]. ROS-induced sperm DNA damage may affect sperm motility and the ability to fertilize an oocyte [14]. Sperm DNA fragmentation (SDF) is irreversible and leads to alteration of sperm function, resulting in infertility [15].

Coenzyme Q10 (CoQ10) is a potent antioxidant that protects sperm against ROS-induced damage [16]. It is quite ubiquitous, with a high amount in sperm mitochondria [17], and has been reported to enhance sperm motility and concentration [8,11]. Furthermore, insufficient CoQ10 levels have been linked to low sperm count and motility, as well as elevated sperm DNA damage [18]. Our recent study conducted on patients with idiopathic oligoasthenospermia (OA) for 12 weeks showed that $\mathrm{CoQ} 10$ therapy substantially enhanced progressive motility, sperm concentration, and seminal fluid CoQ10 concentrations [1]. It also led to an increase in glutathione peroxidase (GPx) levels and total antioxidant capacity (TAC) [1]. Moreover, our recent meta-analysis of three randomized clinical trials on the effect of CoQ10 on semen quality demonstrated beneficial effects of CoQ10 on improving sperm motility, but not on sperm concentration or morphology [18]. The maintenance of testicular scavenging function is normally exerted by intrinsic antioxidants such as GPx, catalase (CAT), and superoxide dismutase (SOD) $[19,20]$.

The present study aimed to investigate the impact of CoQ10 on OS markers and sperm DNA damage in infertile men with idiopathic oligoasthenoteratospermia (OAT) in an attempt to better understand its mechanism of action.

\section{Methods}

\section{Patients}

Fifty patients with idiopathic OAT and 50 fertile men (controls) were recruited at the Fertility Clinic, Hillah, Babyl, Iraq, from July 2018 to January 2019. All patients and controls underwent a comprehensive medical assessment. A prospective controlled study was conducted with a 3-month follow-up. Seven patients dropped out of the study and were therefore excluded. The patients received a daily dose of $200 \mathrm{mg}$ of CoQ10 (in its reduced form as ubiquinol) (America Medic and Science AMS, Woodinville, WA, USA) as a single oral dose for 3 months [21]. Semen analysis, seminal CoQ10 levels, ROS, TAC, CAT, SDF as well as serum hormonal profile (follicle-stimulating hormone $[\mathrm{FSH}]$, luteinizing hormone $[\mathrm{LH}]$, testosterone, and prolactin levels) in patients after therapy were compared with the baseline values for patients and controls (patients were followed-up from September 2018 to February 2019). Sample size calculation was performed using $80 \%$ power and a $5 \%$ level of significance and was 42 for each group. Study approval was obtained from the University of Sumer Local Research Ethical Committee (EC/2018/8879). All partici- pants consented to the study prior to enrollment in the study.

\section{Eligibility criteria}

The fertile controls enrolled in the study had fathered a child in the last 24 months and had normal semen analysis results. The patients had a history of infertility of at least 1 year despite regular unprotected intercourse. OAT was defined according to the World Health Organization (WHO) 2010 criteria. Men with varicocele; genital infection; azoospermia; anatomical abnormalities; testicular injury or surgery; endocrine, renal, hepatic, or other systemic illnesses; smoking; alcohol intake; and recent antioxidant intake were excluded, as were those taking relevant medications and male partners in couples affected by female factor infertility.

\section{Semen analysis}

Semen samples were collected by masturbation following abstinence for 2-3 days. A special wide-mouth container was used to collect semen and incubated at $37^{\circ} \mathrm{C}$ until the semen was liquefied. Semen analysis was then performed within 1 hour following the WHO manual criteria (5th edition, 2010) [22]. Duplicate semen analyses were performed at the beginning and at the end of the study and the average of the two values was used for analysis. The same investigator performed all semen analyses to optimize repeatability.

\section{Measurement of seminal CoQ10 concentrations}

Seminal CoQ10 levels were measured using reverse-phase high-performance liquid chromatography utilizing an ultraviolet light detector at $275 \mathrm{~nm}$, with coenzyme Q9 as an internal standard, and calculated using a published method [23].

\section{Seminal ROS measurement}

Semen samples were centrifuged at 3,000 rpm for 5 minutes to obtain seminal plasma and then were stored at $-20^{\circ} \mathrm{C}$. A manual method was used for ROS measurement as previously described by Venkatesh et al. [24]. To $400 \mu \mathrm{L}$ of liquefied neat semen, $10 \mu \mathrm{L}$ of luminol (5-amino-2,3,-dihydro-1,4-phthalazinedione; Sigma, St. Louis, MO, USA), prepared as $5 \mathrm{mM}$ stock in dimethyl sulfoxide (DMSO), was added. Ten microliters of $5 \mathrm{mM}$ luminol in DMSO served as a blank, while $25 \mu \mathrm{L}$ of $\mathrm{H}_{2} \mathrm{O}_{2}$ with $10 \mu \mathrm{L}$ of luminol was used as a positive control. Luminol-dependent chemiluminescence served as indicator of ROS levels.

\section{Measurement of seminal TAC and CAT}

TAC was estimated with a colorimetric method using a total antioxidant capacity assay kit (\#E-BC-K136; Elabscience, Houston, TX, USA). Seminal plasma CAT activity was assessed using a CAT assay kit (\#E-BC-K031, Elabscience), following the protocol recommended by 
the manufacturer.

\section{Sperm chromatin dispersion test}

Sperm chromatin dispersion was tested using the Halosperm kit (Halotech DNA, Madrid, Spain). The principle of the test is that, after acid denaturation and removal of nuclear proteins, sperm with SDF do not exhibit the halo of dispersed DNA loops that is observed in sperm without SDF. The nucleoids from spermatozoa with SDF show no dispersion halo or a minimal halo. Bright-field microscopy with Diff-Quik staining was utilized to examine the halos. SDF, defined as the percentage ratio of sperm with SDF to the total spermatozoa, was calculated using previously published methods $[1,25]$.

\section{Hormonal assays}

Blood samples ( $5 \mathrm{~mL}$ ) were collected using venipuncture in clean plain labeled tubes, allowed to clot, and centrifuged at 3,000 rpm for 10 minutes for analysis of hormones. Serum FSH, LH, testosterone, and prolactin levels were measured using enzyme-linked fluorescent assays (mini-VIDAS; Biomerieux, Lyon, France).

\section{Statistical analysis}

IBM SPSS ver. 24 (IBM Corp., Armonk, NY, USA) was used for data analysis. The results were expressed as mean \pm standard deviation. The normality of the data distribution was assessed using the Kolmogorov-Smirnov test. One-way analysis of variance was used to compare mean values between subgroups. Pearson correlation coefficients were applied to assess the correlations of seminal fluid parameters with CoQ10 levels and SDF. For all tests, a $p$-value lower than 0.05 was considered to indicate statistical significance.

\section{Results}

The mean age of the control participants and patients was 34.2 \pm 13.4 and $31.3 \pm 12.6$ years, respectively, and the mean duration of infertility in the patients was $7.1 \pm 4.8$ years. CoQ10 therapy in patients with idiopathic OAT significantly increased the total motility $(p<0.01)$, progressive motility $(p<0.05)$, and sperm concentration $(p<0.05)$ compared with the baseline (Table 1). Treatment with CoQ10 increased progressive motility from $26.5 \% \pm 10.8 \%$ to $32.6 \% \pm 15.1 \%$. Sperm concentration also increased from $11.2 \pm 6.4$ to $13.3 \pm 8.6 \mathrm{million} / \mathrm{mL}$ following treatment with $\mathrm{CoQ} 10$, and total motility also rose from $36.1 \% \pm 10.8 \%$ to $44.2 \% \pm 18.5 \%$, with a high level of significance when compared with other sperm parameters.

The results also showed that the seminal antioxidant status was significantly lower in infertile patients than in controls (CoQ10, $p<0.05$; CAT, $p<0.001$; and TAC, $p<0.01$ ), but ROS levels were significantly higher than in controls $(p<0.001)$ (Table 2). Moreover, treatment with CoQ10 substantially enhanced CAT $(p<0.001)$, TAC $(p<0.01)$, and seminal CoQ10 $(p<0.001)$ levels, while decreasing total ROS levels $(p<0.05)$ in patients with idiopathic OAT. The comparison between fertile controls and idiopathic OAT patients showed that SDF was significantly higher in patients $(p<0.001)$, and was significantly reduced by CoQ10 therapy $(p<0.001$ ) (Table 2$)$.

FSH $(p<0.001)$, LH $(p<0.001)$, and prolactin levels $(p<0.01)$ were significantly higher in infertile patients than in controls. Following CoQ10 treatment, LH levels increased significantly in infertile patients compared with their baseline values $(p<0.05)$ (Figure 1). The SDF of infertile patients was negatively correlated with total sperm motility $(r=-0.62, p=0.001)$. Furthermore, total sperm motility $(r=0.56, p=0.005)$ was positively associated with CoQ10 levels $(r=0.23, p=0.12)$ in infertile patients (Table 3).

\section{Discussion}

CoQ10 is an essential antioxidant present nearly in all body tissues. It is also present in sperm mitochondria, where it plays a critical role in cellular respiration and energy generation [26]. CoQ10 is also involved in the inhibition of superoxide formation, protecting against

Table 1. Comparison of semen parameters in fertile men and infertile patients before and after treatment with CoQ10

\begin{tabular}{|c|c|c|c|}
\hline \multirow{2}{*}{ Demographic characteristics and semen parameter } & \multirow{2}{*}{ Fertile men $(n=50)$} & \multicolumn{2}{|c|}{ Infertile patients $(n=50)$} \\
\hline & & Before CoQ10 & After CoQ10 \\
\hline Age (yr) & $34.2 \pm 13.4$ & $31.3 \pm 12.6$ & - \\
\hline Infertility duration (yr) & - & $7.1 \pm 4.8$ & - \\
\hline Volume (mL) & $3.5 \pm 1.4$ & $3.48 \pm 1.7$ & $3.7 \pm 1.8$ \\
\hline Concentration (million/mL) & $61.3 \pm 34.6$ & $11.2 \pm 6.4^{\mathrm{a})}$ & $13.3 \pm 8.6^{c)}$ \\
\hline Progressive motility (\%) & $57.8 \pm 12.2$ & $26.5 \pm 10.8^{\mathrm{a})}$ & $32.6 \pm 15.1^{\mathrm{a}), \mathrm{c}}$ \\
\hline Total motility (\%) & $79.1 \pm 15.0$ & $36.1 \pm 10.8^{\mathrm{a})}$ & $44.2 \pm 18.5^{\mathrm{b}), \mathrm{c}}$ \\
\hline Normal morphology (\%) & $8.2 \pm 3.6$ & $5.4 \pm 3.2^{\mathrm{a})}$ & $5.7 \pm 2.9^{c)}$ \\
\hline
\end{tabular}

Values are presented as mean \pm standard deviation. CoQ10, coenzyme Q10.

a) $p<0.05$, vs. baseline; ${ }^{\text {b) }} p<0.01$, vs. baseline; ${ }^{\text {c) }} p<0.001$, vs. fertile men. 
Table 2. Seminal plasma CoQ10 levels, oxidative stress markers, and sperm DNA fragmentation in fertile controls and infertile patients before and after the administration of CoQ10

\begin{tabular}{|c|c|c|c|}
\hline \multirow{2}{*}{ Variable } & \multirow{2}{*}{ Fertile men $(n=50)$} & \multicolumn{2}{|c|}{ Infertile patients $(n=50)$} \\
\hline & & Before CoQ10 & After CoQ10 \\
\hline CoQ10 level (ng/mL) & $56.7 \pm 37.4$ & $41.4 \pm 29.3^{c)}$ & $76.2 \pm 26.7^{b), d)}$ \\
\hline $\mathrm{ROS}\left(\times 10^{4} \mathrm{RLU} / \mathrm{min} / 20\right.$ million spermatozoa) & $0.08 \pm 0.06$ & $4.3 \pm 1.6^{\mathrm{d})}$ & $3.3 \pm 1.5^{\mathrm{a}, \mathrm{d})}$ \\
\hline $\mathrm{TAC}(\mathrm{mmol} / \mathrm{L})$ & $1.4 \pm 0.25$ & $0.9 \pm 0.44^{\mathrm{d})}$ & $1.2 \pm 0.51^{b), c)}$ \\
\hline Catalase (U/mL) & $15.54 \pm 3.12$ & $10.6 \pm 2.8^{\mathrm{d})}$ & $12.4 \pm 2.61^{\mathrm{a}, \mathrm{d})}$ \\
\hline Sperm DNA fragmentation (\%) & $16.4 \pm 4.7$ & $38.6 \pm 7.9^{\mathrm{d})}$ & $34.5 \pm 9.3^{\mathrm{a}), \mathrm{d})}$ \\
\hline
\end{tabular}

Values are presented as mean \pm standard deviation.

CoQ10, coenzyme Q10; ROS, reactive oxygen species; TAC, total antioxidant capacity.

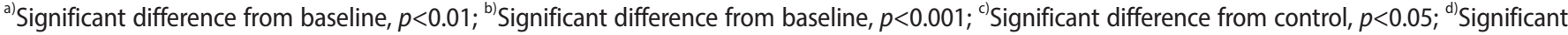
difference from control $p<0.001$.

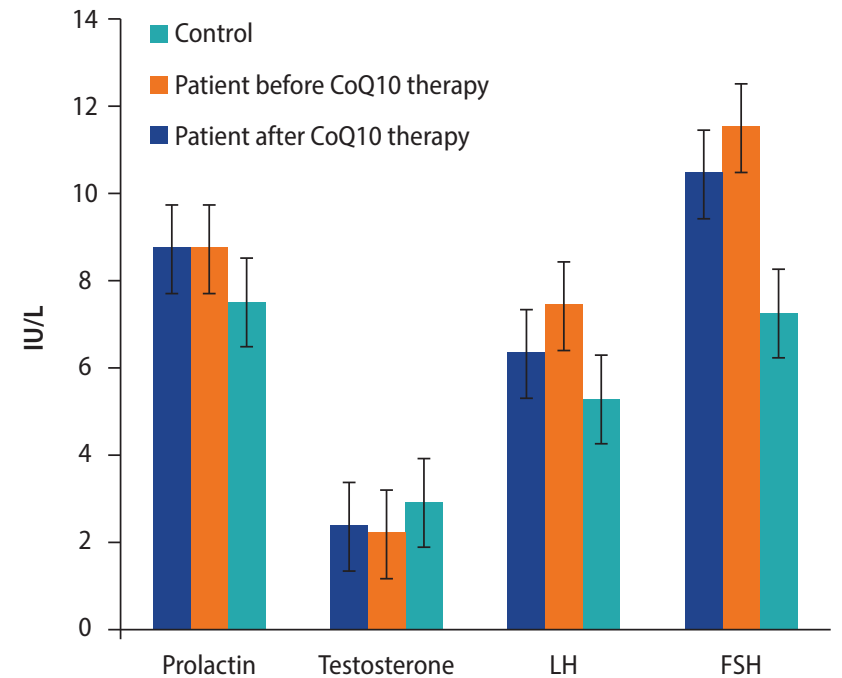

Figure 1. Hormonal profiles of controls and infertile patients with oligoasthenoteratospermia before and after coenzyme Q10 (CoQ10) therapy. LH, luteinizing hormone; FSH, follicle-stimulating hormone.

Table 3. Correlation of SDF and CoQ10 levels with sperm parameters in infertile patients with idiopathic oligoasthenoteratozoospermia

\begin{tabular}{lccc}
\hline \multirow{2}{*}{ Variable } & \multicolumn{3}{c}{$r(p$-value) } \\
\cline { 2 - 4 } & Concentration & Total motility & Normal morphology \\
\hline SDF & $-0.09(0.31)$ & $-0.62(0.001)$ & $-0.18(0.16)$ \\
CoQ10 & $0.23(0.12)$ & $0.56(0.005)$ & $0.14(0.22)$ \\
\hline
\end{tabular}

SDF, sperm DNA fragmentation; CoQ10, coenzyme Q10.

OS-induced sperm damage [27]. OS-mediated disruptions in fertility parameters entail sperm DNA and cell membrane damage [2].

The role of $\mathrm{CoQ} 10$ in cellular respiration and energy generation underscores its usefulness as an antioxidant [28]. In our previous studies, we found that CoQ10 at the dose of $200 \mathrm{mg} /$ day for 3 months improved semen quality and antioxidant status in infertile patients with idiopathic OAT, but the underlying mechanism by which CoQ10 improves fertility parameters are unknown $[1,15]$. In the present study, we found that CoQ10 therapy in patients with id- iopathic OAT significantly improved sperm parameters and antioxidant status, while decreasing OS markers and SDF.

It has been widely reported that SDF is a critical factor that causes male infertility [29]. Thus, the results of the present study may indicate that at the molecular level, CoQ10 acts to ameliorate sperm DNA damage and mitigates OS, thereby leading to improvements in sperm parameters. Similar observations have been published by Suliga and Gluszek [2], who showed that CoQ10 concentrations in seminal plasma were linearly associated with sperm count and motility. Moreover, in a case-control study of 65 idiopathic OA patients, CoQ10 therapy significantly increased progressive motility, GPx levels, sperm concentration, seminal fluid CoQ10 concentration, total motility, and TAC compared with the control group consisting of 45 healthy men [1]. The study concluded that CoQ10 supplementation for 12 weeks led to improvements in OS markers, enhancement of semen parameters, and reduction of SDF in infertile patients with idiopathic OA [1]. Another study conducted in 212 infertile patients with idiopathic OAT, who were on $300 \mathrm{mg}$ of oral CoQ10 for 182 days, showed substantial increases in sperm motility and concentration after CoQ10 treatment [14]. A similar study showed that CoQ10 therapy (200-300 mg per day) could significantly increase sperm motility and concentration [30]. CoQ10 therapy has also been reported to improve SOD and CAT levels, as well as sperm parameters [14]. A meta-analysis conducted by Lafuente et al. [26], also showed that supplementation with CoQ10 enhanced sperm parameters. Furthermore, our recent meta-analysis of three randomized clinical trials demonstrated that CoQ10 improved sperm motility [18]. In a non-controlled trial [31] in 287 infertile patients with idiopathic OAT, it was found that CoQ10 therapy given orally at the dose of $300 \mathrm{mg}$ twice daily for 1 year significantly improved progressive motility, the proportion of normal morphology, and sperm concentration [2].

A controlled trial conducted among 228 idiopathic infertile OAT patients who received CoQ10 supplementation (200 mg daily for 26 weeks) concluded that CoQ10 increased sperm motility, sperm concentration, and morphology [31]. Another controlled trial including 
60 infertile patients with idiopathic OAT treated with CoQ10 (200 mg per day or placebo for 3 months) concluded that there were improvements in semen parameters [32]. The various results from meta-analyses and clinical trials on CoQ10 therapy in men with infertility align with our findings that supplementation with CoQ10 increased seminal CoQ10 levels, sperm motility, and concentration [33]. Generally, controlled clinical trials among men with idiopathic infertility treated with CoQ10 have shown that this treatment leads to a reduction in OS in seminal plasma and lipid peroxidation, as well as an increase in ubiquinol levels and seminal enzymatic antioxidant levels [32].

In a study assessing the effect of 2 doses of CoQ10 on semen quality and OS markers in men with idiopathic OAT, it was found that CoQ10 treatment increased SOD activity, TAC, and CAT activity [34]. CoQ10 inherently inhibits the action of OS by antagonizing any system that increases OS and enhancing systems that could inhibit OS. A prior study showed a strong negative relationship between $\mathrm{CoQ} 10$ and hydrogen peroxide levels [6]. Our results, in agreement with the study by Safarinejad et al. [32], showed that CoQ10 therapy decreased FSH and LH, whereas it increased serum prolactin levels. Hyperprolactinemia is among the causes of hypogonadotropic hypogonadism [35].

A study of 20 infertile patients with high SDF levels treated for 3 months with a preparation containing various antioxidants, including CoQ10, showed that SDF levels significantly decreased and sperm concentration significantly increased [36]. In a study of 20 infertile patients with high SDF levels and low-grade varicocele who were treated for 3 months with a preparation containing CoQ10 and other antioxidants, SDF levels significantly decreased and sperm concentration significantly increased [37]. These results support our finding of a significant decrease in SDF levels when infertile patients with idiopathic OAT were placed on CoQ10 therapy (200 mg/day) [1]. It was also shown that treatment with $\mathrm{CoQ} 10$ substantially enhanced CAT, TAC, and seminal CoQ10 levels in idiopathic OAT, and that total ROS levels decreased when compared with pretreatment values. These findings suggest that CoQ10 therapy could improve sperm parameters in infertile patients with idiopathic OAT.

The present study showed that CoQ10 increased sperm concentration and sperm progressive motility and decreased SDF levels in infertile patients with idiopathic OAT. These findings suggest that CoQ10 therapy improves sperm parameters by reducing OS and OS-induced sperm damage. Therefore, CoQ10 is a potentially useful antioxidant for the treatment of infertile patients with idiopathic OAT.

\section{Conflict of interest}

No potential conflict of interest relevant to this article was reported.

\section{ORCID}

$\begin{array}{ll}\text { Ahmed T Alahmar } & \text { https://orcid.org/0000-0003-2100-5807 } \\ \text { Pallav Sengupta } & \text { https://orcid.org/0000-0002-1928-5048 } \\ \text { Sulagna Dutta } & \text { https://orcid.org/0000-0002-7893-5282 } \\ \text { Aldo E. Calogero } & \text { https://orcid.org/0000-0001-6950-335X }\end{array}$

\section{Author contributions}

Conceptualization, Data curation, Formal analysis, \& Methodology: ATA. Project administration: ATA, PS, AEC. Visualization: all authors. Writing-original draft: ATA. Writing \& review-editing: PS, SD, AEC.

\section{References}

1. Alahmar AT, Calogero AE, Sengupta P, Dutta S. Coenzyme Q10 improves sperm parameters, oxidative stress markers and sperm DNA fragmentation in infertile patients with idiopathic oligoasthenozoospermia. World J Mens Health 2021;39:346-51.

2. Suliga E, Gluszek S. The relationship between diet, energy balance and fertility in men. Int J Vitam Nutr Res 2020;90:514-26.

3. Agarwal A, Mulgund A, Hamada A, Chyatte MR. A unique view on male infertility around the globe. Reprod Biol Endocrinol 2015; 13:37.

4. Ilacqua A, Izzo G, Emerenziani GP, Baldari C, Aversa A. Lifestyle and fertility: the influence of stress and quality of life on male fertility. Reprod Biol Endocrinol 2018;16:115.

5. Esteves SC. Clinical relevance of routine semen analysis and controversies surrounding the 2010: World Health Organization criteria for semen examination. Int Braz J Urol 2014;40:443-53.

6. Alahmar AT. Role of oxidative stress in male infertility: an updated review. J Hum Reprod Sci 2019;12:4-18.

7. Tadros NN, Sabanegh ES. Empiric medical therapy with hormonal agents for idiopathic male infertility. Indian J Urol 2017;33:194-8.

8. Darbandi M, Darbandi S, Agarwal A, Sengupta P, Durairajanayagam D, Henkel R, et al. Reactive oxygen species and male reproductive hormones. Reprod Biol Endocrinol 2018;16:87.

9. Tirabassi G, Vignini A, Tiano L, Buldreghini E, Bruge F, Silvestri S, et al. Protective effects of coenzyme Q10 and aspartic acid on oxidative stress and DNA damage in subjects affected by idiopathic asthenozoospermia. Endocrine 2015;49:549-52.

10. Bentov Y, Hannam T, Jurisicova A, Esfandiari N, Casper RF. Coenzyme Q10 supplementation and oocyte aneuploidy in women undergoing IVF-ICSI treatment. Clin Med Insights Reprod Health 2014:8:31-6.

11. Alahmar AT. The effects of oral antioxidants on the semen of men with idiopathic oligoasthenoteratozoospermia. Clin Exp Reprod 
Med 2018;45:57-66.

12. Dutta $S$, Henkel R, Sengupta P, Agarwal A. Physiological role of ROS in sperm function. In: Parekattil Sijo J, Esteves SC, Agarwal A, editors. Male infertility: contemporary clinical approaches, andrology, ART and antioxidants. Cham: Springer; 2020. p. 337-45.

13. Dutta S, Majzoub A, Agarwal A. Oxidative stress and sperm function: a systematic review on evaluation and management. Arab J Urol 2019;17:87-97.

14. Wright C, Milne S, Leeson H. Sperm DNA damage caused by oxidative stress: modifiable clinical, lifestyle and nutritional factors in male infertility. Reprod Biomed Online 2014;28:684-703.

15. Majzoub A, Agarwal A. Systematic review of antioxidant types and doses in male infertility: benefits on semen parameters, advanced sperm function, assisted reproduction and live-birth rate. Arab J Urol 2018;16:113-24.

16. Alahmar AT, Sengupta P. Impact of coenzyme Q10 and selenium on seminal fluid parameters and antioxidant status in men with idiopathic infertility. Biol Trace Elem Res 2021;199:1246-52.

17. Panner Selvam MK, Agarwal A. A systematic review on sperm DNA fragmentation in male factor infertility: laboratory assessment. Arab J Urol 2018;16:65-76.

18. Vishvkarma R, Alahmar AT, Gupta G, Rajender S. Coenzyme Q10 effect on semen parameters: Profound or meagre? Andrologia 2020;52:e13570.

19. Imamovic Kumalic S, Pinter B. Review of clinical trials on effects of oral antioxidants on basic semen and other parameters in idiopathic oligoasthenoteratozoospermia. Biomed Res Int 2014;2014: 426951.

20. Alahmar AT. The impact of two doses of coenzyme Q10 on semen parameters and antioxidant status in men with idiopathic oligoasthenoteratozoospermia. Clin Exp Reprod Med 2019;46:112-8.

21. Balercia G, Buldreghini E, Vignini A, Tiano L, Paggi F, Amoroso S, et al. Coenzyme Q10 treatment in infertile men with idiopathic asthenozoospermia: a placebo-controlled, double-blind randomized trial. Fertil Steril 2009;91:1785-92.

22. World Health Organization. WHO laboratory manual for the examination and processing of human semen. 5th ed. Geneva: WHO Press; 2010.

23. Li K, Shi Y, Chen S, Li W, Shang X, Huang Y. Determination of coenzyme Q10 in human seminal plasma by high-performance liquid chromatography and its clinical application. Biomed Chromatogr 2006;20:1082-6.

24. Venkatesh S, Shamsi MB, Dudeja S, Kumar R, Dada R. Reactive oxygen species measurement in neat and washed semen: comparative analysis and its significance in male infertility assessment.
Arch Gynecol Obstet 2011;283:121-6.

25. Zaazaa A, Adel A, Fahmy I, Elkhiat Y, Awaad AA, Mostafa T. Effect of varicocelectomy and/or mast cells stabilizer on sperm DNA fragmentation in infertile patients with varicocele. Andrology 2018; 6:146-50.

26. Lafuente R, Gonzalez-Comadran M, Sola I, Lopez G, Brassesco M, Carreras R, et al. Coenzyme Q10 and male infertility: a meta-analysis. J Assist Reprod Genet 2013;30:1147-56.

27. Minamiyama Y, Ichikawa H, Masui T, Kobayashi K, Takemura S, Oka MO, et al. Oral administration of reduced coenzyme Q10 ameliorates the endocrine-disrupting chemical-induced sperm toxicity in rats. Free Radic Biol Med 2016;100:S105-6.

28. Beharry KD, Cai CL, Henry MM, Chowdhury S, Valencia GB, Aranda JV. Co-Enzyme Q10 and n-3 polyunsaturated fatty acid supplementation reverse intermittent hypoxia-induced growth restriction and improved antioxidant profiles in neonatal rats. Antioxidants (Basel) 2017;6:103.

29. Leaver RB. Male infertility: an overview of causes and treatment options. Br J Nurs 2016;25:S35-40.

30. Majzoub A, Agarwal A. Antioxidant therapy in idiopathic oligoasthenoteratozoospermia. Indian J Urol 2017;33:207-14.

31. Safarinejad MR. The effect of coenzyme Q11 supplementation on partner pregnancy rate in infertile men with idiopathic oligoasthenoteratozoospermia: an open-label prospective study. Int Urol Nephrol 2012;44:689-700.

32. Safarinejad MR, Safarinejad S, Shafiei N, Safarinejad S. Effects of the reduced form of coenzyme Q10 (ubiquinol) on semen parameters in men with idiopathic infertility: a double-blind, placebo controlled, randomized study. J Urol 2012;188:526-31.

33. Nadjarzadeh A, Shidfar F, Amirjannati N, Vafa MR, Motevalian SA, Gohari MR, et al. Effect of Coenzyme Q10 supplementation on antioxidant enzymes activity and oxidative stress of seminal plasma: a double-blind randomised clinical trial. Andrologia 2014; 46:177-83.

34. Al-Mosawi AM. The role of different antioxidant agents in human infertility and assisted reproductive techniques. J Nat Sci Res 2016; 6:57-64.

35. Tiseo BC, Gaskins AJ, Hauser R, Chavarro JE, Tanrikut C; EARTH Study Team. Coenzyme Q10 intake from food and semen parameters in a subfertile population. Urology 2017;102:100-5.

36. Huang C, Cao X, Pang D, Li C, Luo Q, Zou Y, et al. Is male infertility associated with increased oxidative stress in seminal plasma? A-meta analysis. Oncotarget 2018;9:24494-513.

37. Ko EY, Sabanegh ES. The role of nutraceuticals in male fertility. Urol Clin North Am 2014;41:181-93. 